\section{Application of Electrodeposition and Etching Forms Stable Metallic Nanogaps}

As research heads toward the age of nanoelectronics, some applications require electrodes with gap sizes on the same order of magnitude as the size of an atom or molecule. Researchers from the Department of Physics at Florida International University have fabricated stable molecular-scale gaps with sub-angstrom precision. As reported in the December 18 issue of Applied Physics Letters, gaps a few nm or less can be fabricated with a $0.5 \AA$ resolution. The gap sizes may be readily increased or decreased by the process of electrodepositioning or etching. The researchers indirectly measured the gap widths by the amplitudes of the electron tunneling current.

The initial calibration setup was comprised of a $\mathrm{Pt}_{0.8} \mathrm{Ir}_{0.2}$ scanning tunneling microscope (STM) tip coated with Apiezon wax and a 100-nm thick gold film on a mica substrate in an electrical feedback circuit. The researchers C.Z. Li, H.X. He, and N.J. Tao narrowed the initial gap, $50 \mathrm{~nm}$ wide, by bathing the STM tip in a $\mathrm{CuSO}_{4}$ and $\mathrm{H}_{2} \mathrm{SO}_{4}$ electrolytic solution. This caused copper to deposit onto the STM tip. They measured the tunneling current continuously while the gap narrowed. In order to produce long-term stable nanogaps, the researchers fabricated a pair of gold electrodes on oxidized silicon. An expected increase in tunneling current as the gap narrowed was observed. The unexpected event, however, was the observation of step-wise changes during this climb. The researchers said that the discrete nature of atoms may have caused this quantization in tunneling current through a process known as structural relaxation. Relaxed structures supply stable energy wells, which provide a predictable means of gap construction. These discrete changes in currents correspond to $0.5 \AA$ changes to the physical widths of the gap.

The researchers further controlled the gap width with a feedback loop through reversibility by electrochemical etching. This is similar to the deposition process except for the reversed electrochemical potential. This method of nanogap fabrication contributes toward the future of nanotechnology due to its precision, stability, and reversibility.

JUNE LAU

\section{Atomic Force Microscope Aids Fabrication Control of Gold Nanowires}

A group of scientists from the National Research Institute for Metals in Japan has developed a technique for controlled growth of gold nanowires. The researchers explained their method as well as their results in measurements of electronic transport in the December 25 issue of Applied Physics Letters. Using an atomic force microscope (AFM) in contact mode with a piezoresistive cantilever coated with gold, they grew the nanowires on top of a Si(111) substrate in ultrahigh vacuum. Prior to $\mathrm{Au}$ deposition the silicon is flashed to $1200^{\circ} \mathrm{C}$ several times until a clear $7 \times 7$ surface reconstruction is observed. The surface is then checked for cleanliness by Auger spectroscopy and scanning tunneling microscopy. The nanowires are then drawn onto the surface with the tip in contact. The gold actually "flows from the tip to the surface, like a fountain pen," said the researchers, while the cantilever is moving on the $\mathrm{Si}(111)$ surface. The resultant wires can be observed under imaging with the AFM in noncontact mode. Depending on the bias voltage applied to the tip, the contrast of the image changes. This effect is due to a difference in the Fermi level that causes electrons to flow from the Si substrate to the gold wire, charging it negatively. Consequently, it is possible to establish the metallic nature of these gold wires.

The line width of the wires depends almost exclusively on the characteristics of the cantilever tip, given that its velocity is less than $50 \mathrm{~nm} / \mathrm{s}$. The researchers obtained wires with a line width of $4 \mathrm{~nm}$ and lengths of $7 \mu \mathrm{m}$.

The researchers used four joint evaporated Ag electrodes to measure the conductance in the gold wires at liquid helium temperatures. The $I-V$ curve for an applied bias voltage of $\pm 100 \mathrm{mV}$ is linear, corroborating the metallic behavior of the nanowires, although for larger bias voltages the $I-V$ curve is nonlinear. According to the researchers, this is probably caused by the instability of the wires fabricated over the Ag electrodes, or by effects of the Si substrate.

This method has the advantage of great control of the cantilever tip, which means a potential for fabrication of nanometer devices at industrial level, especially if an array of cantilevers is developed.

SIARI S. SOSA

\section{Electron Transfer Produces $n$-Type Colloidal Semiconductor Nanocrystals}

M. Shim and P. Guyot-Sionnest at the James Franck Institute, University of Chicago have produced $n$-type colloidal semiconductor nanocrystals of CdSe, $\mathrm{CdS}$, and $\mathrm{ZnO}$ by using electron transfer techniques similar to those used with conducting polymers. The $n$-type character of the nanocrystals is maintained for several hours (in CdS) and for several days (in $\mathrm{ZnO}$ ) at room temperature. According to the researchers, this is the first time that electrons have been successfully placed in the lowest unoccupied quantum-confined orbital (LUQCO) of strongly confined colloidal semiconductor nanocrystals without optical creation of an electron hole pair.

As reported in their article published in the October 26 issue of Nature, the scientists added $\sim 50 \mathrm{mM}$ sodium biphenyl into a $\sim 0.1 \mathrm{mM}$ dried and degassed solution of CdSe nanocrystals in hepthamethylnonane. Electron transfer from the sodium biphenyl to the nanocrystal produced $n$-CdSe nanocrystals. The researchers measured the absorption spectra of CdSe nanocrystals using infrared spectroscopy. Shim and Guyot-Sionnest observed a strong peak infrared absorption at $0.3 \mathrm{eV}$. In these nearly spherical nanocrystals, the infrared transition indicates the presence of $n$-type nanocrystals and arises when the electron is excited from the lowest conduction band state $1 S_{e}$ to the next state $1 P_{e}$, similar to a hydrogen atom. To determine the different features of electron transitions from the LUQCO to the next higher state, the researchers measured the absorption spectra of various sizes of $n$-type CdSe nanocrystals and compared their results to previous research. When CdSe is subjected to various capping groups, they said, electrons still moved to quantum-confined states producing $n$-type semiconductor nanocrystals.

The researchers further confirmed their results by measuring the infrared absorption spectra of $n$-type $\mathrm{CdS}$ and $\mathrm{ZnO}$ nanocrystals along with CdSe, and observed the time decay of each absorption spectrum at room temperature. They found that the infrared absorption in $\mathrm{ZnO}$ nanocrystals takes a much longer time to decay than the corresponding absorption in n-type CdSe and CdS nanocrystals. According to the report, the stability of the $n$-type semiconductor nanocrystals is largely dependent on the reduction potential of the nanocrystals and the temperature of the surroundings. Since the electron transfer approach requires the injection of electrons into the LUQCO, eliminating electron traps in the bandgap of the colloidal semiconductor nanocrystals could also enhance stability of electrons in the quantum-confined states.

In terms of future applications, the researchers proposed that as methods are devised to control the electron injection by an external potential or current, novel electrochromic materials would be 